\title{
Electronic autoionization and vibrational-state distributions in resonant multiphoton ionization of $\mathbf{H}_{2}$
}

\author{
S. N. Dixit \\ Theoretical Atomic and Molecular Physics Group, Lawrence Livermore National Laboratory, L-446 \\ Livermore, California 94550 \\ D. L. Lynch \\ Los Alamos National Laboratory, Los Alamos, New Mexico 87545 \\ and Department of Chemistry, University of Nevada, Reno, Nevada 89557 \\ B. V. McKoy \\ Noyes Laboratory of Chemical Physics, California Institute of Technology, Pasadena, California 91125
}

A. U. Hazi

Theoretical Atomic and Molecular Physics Group, Lawrence Livermore National Laboratory, L-446

Livermore, California 94550

(Received 28 December 1988)

\begin{abstract}
We investigate the effects of electronic autoionization on the vibrational branching ratios in resonant multiphoton ionization of $\mathrm{H}_{2}$. Ab initio calculations are performed to obtain the vibrational branching ratios for $(3+1)$ resonant-enhanced multiphoton ionization (REMPI) of $\mathrm{H}_{2}$ via the $C^{1} \Pi_{u}$ state. Our calculations include the effects of the dissociative ${ }^{1} \Pi_{g}\left(1 \sigma_{u} 1 \pi_{u}\right)$ autoionizing state and properly account for the interference between the direct and the indirect (autoionization) channels. We find that the direct and indirect amplitudes are comparable for excitation via the higher $\left(v_{i} \geq 2\right)$ vibrational levels of the $C$ state. Autoionization greatly enhances the branching ratios for $\Delta v \neq 0$ transitions. These calculations underscore the necessity for a proper treatment of both the direct and indirect contributions in understanding the REMPI of molecules via autoionizing states.
\end{abstract}

Resonant-enhanced multiphoton ionization (REMPI), combined with high-resolution photoelectron spectroscopy, is a powerful probe of the photoionization dynamics of atomic and molecular excited states. Recently, several experimental groups have used this technique to demonstrate non-Franck-Condon behavior of vibrational branching ratios, effects of Rydberg-valence mixing, shape resonances, and rotational, vibrational, and electronic autoionization in small molecules. ' In one of the first experimental studies of this nature, Pratt, Dehmer, and Dehmer $^{2}$ measured the vibrational branching ratios resulting from $(3+1)$ REMPI of $\mathrm{H}_{2}$ via the $C^{1} \Pi_{u}$ state. Due to the Rydberg nature of the $C^{1} \Pi_{u}$ state, one expects direct ionization to preserve the vibrational quantum number $\left(v_{i}\right)$ of the resonant state. ${ }^{3}$ The experimental photoelectron spectra indeed showed large $\Delta v=0 \quad\left(\Delta v=v_{+}-v_{i}\right)$ peaks. However, the intensities of the $\Delta v \neq 0$ peaks differed from Franck-Condon predictions with increasing deviations for excitations through higher vibrational levels of the $C^{1} \Pi_{u}$ state. $^{2}$ In our earlier attempts ${ }^{4}$ to understand the origin of the observed non-Franck-Condon behavior, we included variations of the photoionization matrix elements with both electron kinetic energy $\left(\varepsilon_{k}\right)$ and internuclear separation $(R)$. While the calculated and measured branching ratios agreed well for ionization via the $v_{i}=0-2$ levels, significant differences remained for $v_{i}=3-6$. Subsequent measurements of photoelectron angular distributions ${ }^{5}$ and angle-integrated branching ra- $\operatorname{tios}^{6,7}$ also indicated similar discrepancies for $\Delta v \neq 0$ peaks for REMPI through $v_{i} \geq 2$ states.

Recently, Chupka ${ }^{8}$ and Hickman ${ }^{9}$ have proposed that the anomalous vibrational distributions in the $(3+1)$ REMPI photoelectron spectra arise from electronic autoionization via the doubly excited ${ }^{1} \Pi_{g}\left(1 \sigma_{u} 1 \pi_{u}\right)$ state. Using a previously computed potential-energy curve, ${ }^{10}$ Chupka has argued that the perturbations should be strongest around $v_{i} \approx 4$. Autoionization through doubly excited electronic states adds an indirect contribution to the ionization process which interferes with the direct photoionization amplitude. , $^{8,11}$ A quantitative treatment of such interference must include contributions from both the direct and resonant channels. Model calculations by Hickman, ${ }^{9}$ analyzing the effects of autoionization on the ionic vibrational-state distributions, did not include the contribution from direct ionization. The adequacy of this approximation has not been assessed on the basis of detailed first-principle calculations. In fact, the measured photoelectron angular distributions ${ }^{5}$ indicate that the direct contribution is present even for $v_{i} \geq 4$.

In this paper, we examine the influence of the doubly excited ${ }^{1} \Pi_{g}$ autoionizing state on the vibrational branching ratios in $(3+1)$ REMPI of $\mathrm{H}_{2}$ via the $C^{1} \Pi_{u}$ state. Although the ${ }^{1} \Sigma_{g}^{+}\left(1 \sigma_{u}^{2}\right)$ state is also energetically accessible, the dominant contribution to autoionization should come from the ${ }^{1} \Pi_{g}$ state. ${ }^{12}$ We present the results of $a b$ initio calculations which include the effects of autoioniza- 
tion and interference between direct and autoionization channels. The required continuum state and bound-state wave functions were obtained using the Schwinger variational method and the configuration interaction method, respectively.

The dissociative nature of the ${ }^{1} \Pi_{g}$ autoionizing state introduces competition between dissociation and autoionization. ${ }^{8,9}$ We analyze this dissociative autoionization process by generalizing the resonant scattering theory, ${ }^{13-16}$ which has been used previously for studying the dissociative attachment of low-energy electrons, to include photoexcitation from the $C{ }^{1} \Pi_{u}$ state. Our model is similar to the theory developed by Sobolewski and Domcke, ${ }^{17}$ who employ a projection operator formalism to study the effects of both vibrational and electronic autoionization on molecular photoionization. It can be shown that the wave function of the dissociating nuclei having a total energy $E$ is described by

$$
\begin{aligned}
& \left(-\frac{1}{2 \mu} \frac{d^{2}}{d R^{2}}+V(R)-E\right) \xi_{v_{i}^{\prime}}(R)+\sum_{v_{+}} \chi_{v_{+}}(R) \iint_{\mathrm{p}} d \varepsilon_{k} \frac{H_{Q P}\left(\varepsilon_{k}, R\right)}{\left(E-\varepsilon_{k}-E_{v_{+}}\right)} \int d R^{\prime}\left\langle\chi_{v_{+}}\left(R^{\prime}\right)\left|H_{P Q}\left(\varepsilon_{k}, R^{\prime}\right)\right| \xi_{v_{i}}\left(R^{\prime}\right)\right\rangle \\
& \left.-h\left(E-E_{v_{+}}\right) i \pi H_{Q P}\left(\varepsilon_{k}^{+}, R\right) \int d R^{\prime}\left\langle\chi_{v_{+}}\left(R^{\prime}\right)\left|H_{P Q}\left(\varepsilon_{k}^{+}, R^{\prime}\right)\right| \xi_{v_{i}}\left(R^{\prime}\right)\right\rangle\right) \\
& =H_{Q S}(R) \chi_{v_{i}}(R)+\sum_{v_{+}} \chi_{v_{+}}(R) \iint_{\mathrm{p}} d \varepsilon_{k} \frac{H_{Q P}\left(\varepsilon_{k}, R\right)}{\left(E-\varepsilon_{k}-E_{v_{+}}\right)} \int d R^{\prime}\left\langle\chi_{v_{+}}\left(R^{\prime}\right)\left|H_{P S}\left(\varepsilon_{k}, R^{\prime}\right)\right| \chi_{v_{i}}\left(R^{\prime}\right)\right\rangle \\
& \left.-h\left(E-E_{v_{+}}\right) i \pi H_{Q^{P}}\left(\varepsilon_{k}^{+}, R\right) \int d R^{\prime}\left\langle\chi_{v_{+}}\left(R^{\prime}\right)\left|H_{P S}\left(\varepsilon_{k}^{+}, R^{\prime}\right)\right| \chi_{v_{i}}\left(R^{\prime}\right)\right\rangle\right) \text {, }
\end{aligned}
$$

where $V(R)$ denotes the potential curve of the autoionizing state, $\mu$ the reduced mass of nuclei, and $\chi_{v_{i}}$ and $\chi_{v_{+}}$the vibrational wave functions of the $C^{1} \Pi_{u}$ state and the ion $X^{2} \Sigma_{g}^{+}$state, respectively. The energies of the vibrational levels of these electronic states are denoted by $E_{v_{i}}$ and $E_{c^{+}}$, respectively. In Eq. (1), $H_{P Q}\left(\varepsilon_{k}, R\right)$ denotes the matrix element coupling the autoionizing state ( $Q$ space) and the photoelectron continuum ( $P$ space) at electron kinetic energy $\varepsilon_{k}\left(E-E_{v_{+}}+\varepsilon_{k}^{+}\right) . H_{P S}$ and $H_{Q S}$ denote the photoexcitation matrix elements from the $C^{1} \Pi_{u}$ state $(S$ space) to the continuum and the autoionizing level, respectively. The symbol $\int_{\mathrm{p}}$ denotes a principal value integral, and the Heaviside function $h\left(E-E_{v_{+}}\right)$ensures that the sums of the "on-shell" terms over $v+$ include only energetically open vibrational states of the molecular ion. The effect of autoionization on the dissociative wave function is represented through the nonlocal, or integral, operators on the left-hand side. The right-hand side of Eq. (1) describes the two possible ways of photopumping the molecule from the $C^{1} \Pi_{u}$ state to the autoionizing state: the first term represents direct photoexcitation, and the second an indirect mechanism in which the molecule is excited into a continuum state, followed by a resonant capture (inverse autoionization) of the outgoing electron to form the autoionizing state.

Equation (1) is considerably more general than that used by Hickman, ${ }^{9}$ who neglected both the principal value term on the left-hand side, and the indirect excitation terms (with $H_{Q P}$ and $H_{P S}$ ) on the right-hand side. Our result is correct to infinite order in the coupling $H_{P Q}$ and to first order in the photon-molecule couplings $\left(H_{Q S}\right.$ and $\left.H_{P S}\right)$. The details of the derivation of Eq. (1) will be published elsewhere.

With $\xi_{v_{i}}$ determined from Eq. (1), the cross section for photoionization into a particular ionic vibrational state $v_{+}^{0}$ can be written as

$$
\sigma_{v_{+}^{0}+} \propto\left|\left\langle\chi_{v_{+}^{0}+}\left|H_{P S}\right| \chi_{v_{i}}\right\rangle+\left\langle\chi_{v_{+}^{0}+}\left|H_{P Q}\right| \xi_{v_{i}}\right\rangle\right|^{2},
$$

which clearly exhibits the interference between the direct and indirect channels.

For the wave functions of the resonant $C{ }^{1} \Pi_{u}$ and the autoionizing ${ }^{1} \Pi_{g}$ states, we used the " $g$ " atomic basis set of Guberman ${ }^{10}$ and carried out full configuration-interaction (CI) calculations with the $\left(7 \sigma_{g} 7 \sigma_{u} 4 \pi_{u} 4 \pi_{g}\right)$ ionic molecular-orbital basis set. The photoelectron wave functions were obtained using the Schwinger variational techniques. ${ }^{18}$ Particular care was given to ensure that the relative phases, as a function of internuclear distance, were consistent for the various electronic wave functions. Our calculated autoionization widths are in good agreement with those of Tennyson and Noble ${ }^{19}$ and Collins and Schneider. ${ }^{20}$ The required vibrational wave functions were calculated using Cooley's method. ${ }^{21}$

The nonlocal nature of the principal value term on the left-hand side of Eq. (1) complicates the calculation of the dissociative wave function $\xi_{v_{i}}(R)$. Here, we simplify the solution of Eq. (1) by approximating the nonlocal shift term by its local value

$$
S(E, R)=\int_{\mathrm{p}} d \varepsilon_{k} \frac{\left|H_{Q P}\left(\varepsilon_{k}, R\right)\right|^{2}}{\left(E-\varepsilon_{k}-\bar{E}\right)},
$$

with $\bar{E}=E_{v_{+}}=v_{i}$. This introduces some uncertainty in the effective potential curve of the autoionizing state, but we have verified that the calculated branching ratios are not extremely sensitive to small changes in this parameter. The final equation resulting from Eq. (1) with this approximation is solved using the separable kernel method $^{14-16}$ retaining ten vibrational states in the summation over $v+$. 
TABLE I. Photoionization cross sections (in units $10^{-18}$ $\mathrm{cm}^{2}$ ) out of the $J=1, M=1$ level of the $C^{1} \Pi_{u}$ state, summed over the ionic vibrational states.

\begin{tabular}{ccccc}
\hline \hline$v_{i}$ & Direct & Indirect & Total & Indirect $^{\mathrm{a}}$ \\
\hline 0 & 7.802 & 0.0445 & 7.314 & 0.0464 \\
1 & 6.823 & 0.130 & 6.265 & 0.143 \\
2 & 5.970 & 0.478 & 5.501 & 0.542 \\
3 & 5.260 & 2.046 & 6.040 & 2.45 \\
4 & 4.621 & 4.371 & 8.528 & 6.60 \\
5 & 3.977 & 2.584 & 4.420 & \\
6 & 3.609 & 2.279 & 6.109 & \\
\hline \hline
\end{tabular}

${ }^{a}$ Reference 9.

The effects of alignment on the REMPI vibrational branching ratios and on the photoelectron angular distributions are treated using the framework described earlier. ${ }^{22}$ Based on the arguments given in Ref. 4, $(3+1)$ REMPI via the $Q(1)$ branch of the $C^{1} \Pi_{u}$ state of $\mathrm{H}_{2}$ is determined by the ionization rate out of the $J=1, M=1$ level of the $\Pi^{-}$component. Symmetry considerations also imply that the ${ }^{1} \Pi_{g}$ autoionizing state couples only to the $1 \sigma_{g} k \pi_{g}$ continuum. The $k \sigma_{g}$ and $k \delta_{g}$ continua are unaffected and contribute only to the direct channel.

Table I presents the total cross sections for ionization of the $J=1, M=1$ level of the $C^{1} \Pi_{u}^{-}$state summed over the rotational and vibrational levels of the $\mathrm{H}_{2}{ }^{+} X^{2} \Sigma_{g}^{+}$state. The autoionization contribution is small for ionzation via $v_{i} \leq 2$ levels. For $v_{i}=3-6$, however, the direct and in- direct contributions are comparable. The peaking of the indirect contribution around $v_{i} \approx 4$ is consistent with the work of Chupka ${ }^{8}$ and with the observed trends, ${ }^{5,6,23}$ although it is by no means small compared to the direct contribution for $v_{i}>4$. Further, note the changing role of interference between the direct and indirect channels with increasing $v_{i}$. Our indirect cross sections agree with those of Ref. 9 for low $v_{i}(\leq 2)$ but differ increasingly for $v_{i}>2$. This may be due to the assumption, in Ref. 9, that the $R$ dependence of the $C^{1} \Pi_{u}-{ }^{1} \Pi_{g}$ transition moment $\left(H_{Q S}\right)$ is $-R / 2$. This approximation, based on the $1 \sigma_{g^{-}}$ $1 \sigma_{u} \mathrm{H}_{2}{ }^{+}$transition moment ${ }^{24}$ and the spectator nature of the Rydberg $1 \pi_{u}$ orbital, is adequate at small $R$ but breaks down at large $R$ due to correlation effects. Our CI calculations show that the dipole transition moment is smaller than $R / 2$ at large internuclear distances; e.g., for $R=4.0$ a.u., its value is 1.4 a.u. Measurements of the absolute cross sections for ionization out of various vibrational levels would be useful in understanding these trends.

In Fig. 1, we compare our calculated vibrational branching ratios with the experimental values for $(3+1)$ REMPI via the $v_{i}=0-6$ levels of the $C^{1} \Pi_{u}$ state. Both theoretical and experimental values are for photoelectron detection along the direction of laser polarization $(\theta$ $=0^{\circ}$ ). Unlike our earlier comparisons, we normalize the vibrational branching ratios such that their sum is unity. We find that autoionization has a minor effect for excitation out of $v_{i}=0,1$ in agreement with the conclusions in Ref. 9. Generally, autoionization seems to transfer inten-
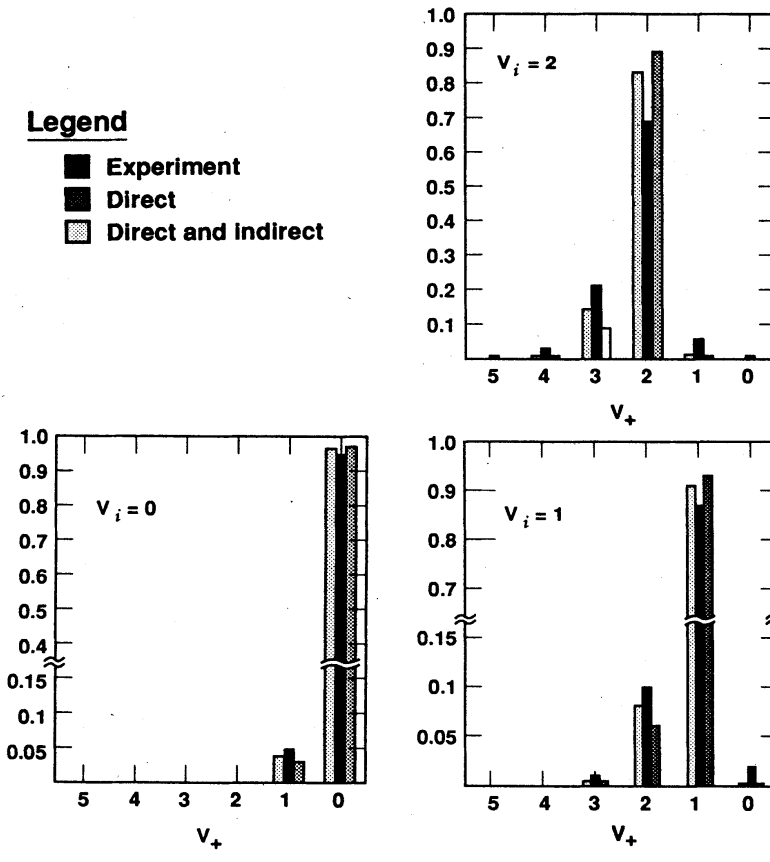
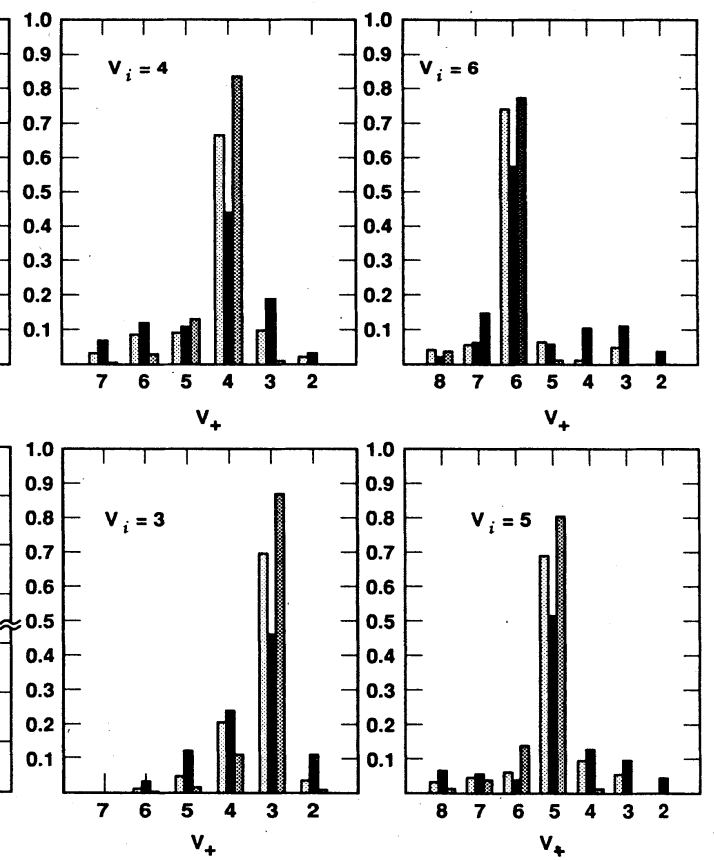

FIG. 1. Vibrational branching ratios in $(3+1)$ REMPI of $\mathrm{H}_{2}$ via the $Q(1)$ line of the $C^{1} \Pi_{u}$ state. Experimental and theoretical results are for detection along the laser polarization axis $\left(\theta=0^{\circ}\right)$. Central dark bars: experimental data for $v_{i}=0-4$, from Table $I$ of Ref. 5 and for $v_{i}=5$ and 6 from Fig. 4 of Ref. 23; bars to the right of central ones: calculations with direct channel only; bars to the left of central ones: calculations with direct and indirect channels. All branching ratios are normalized such that the sum total equals unity. 
sity from the $v_{+}=v_{i}$ peaks into the $v_{+} \neq v_{i}$ peaks. Inclusion of autoionization clearly improves the agreement between theory and experiment for $\Delta v \neq 0$ transitions. This is particularly dramatic for the $v+<v_{i}$ peaks which were negligible in the absence of autoionization. The peaking of the autoionization contribution around $v_{i} \approx 4$ in Table $I$ is also reflected in the increasing difference between the "direct" and "total" branching ratios for the $\Delta v=0$ transitions up to $v_{i}=4$ followed by a decrease thereafter. These results also demonstrate that the direct ionization contribution is significant for all levels considered $\left(v_{i} \leq 6\right)$.

Comparison of the calculated and measured $v_{+}=v_{i}$ branching ratios shows interesting dependence on $v_{i}$. The deviation between theory and experiment, while small for $v_{i}=0,1$, increases for $v_{i}>2$. For $v_{+}=v_{i}$, the calculated branching ratios decrease with increasing $v_{i}$ while the experimental ratios first decrease up to $v_{i}=4$ and increase thereafter. At present we do not have an explanation for this discrepancy. Possible reasons for these differences include effects such as the saturation of the final step and contribution from higher-lying autoionizing levels. ${ }^{10,19}$

The present results clearly demonstrate that both direct and indirect contributions have comparable influence on the REMPI dynamics in $\mathbf{H}_{2}$. Furthermore, experimental photoelectron angular distributions ${ }^{5}$ suggest that the diagonal $(\Delta v-0)$ peaks have large contributions from direct ionization. Therefore, the procedure of Ref. 9 of normalizing the calculated indirect branching ratios to the measured values at the $\Delta v=0$ peak is not justified.

In summary, we have studied the role of electronic autoionization on the vibrational branching ratios in a $(3+1)$ REMPI via the $C^{1} \Pi_{u}$ state of $H_{2}$. Our calculations properly account for the interference between the direct and the indirect channels and clearly illustrate the importance of autoionization in enhancing ${ }^{8,9}$ the $v+\neq v_{i}$ branching ratios. The direct and autoionization channels are seen to be of comparable magnitude for $v_{i} \geq 2$. In the present work, we have included only the lowest ${ }^{1} \Pi_{g}$ autoionizing state of $\mathrm{H}_{2}$. Results of the investigation of the contribution of other autoionizing states to the REMPI process will be presented in the future.

Generally, doubly excited autoionizing states are expected to lie close to the ionization threshold, particularly at large internuclear distances. These states will perturb the REMPI spectra of a wide range of molecules (e.g., $\mathrm{O}_{2}$, $\mathrm{Cl}_{2}$, etc.) for ionization via higher vibrational levels. The presence of autoionization also introduces serious complications in the extraction of state populations from REMPI signals and in the use of REMPI for the production of ions in specific vibrational levels. Studies of the type presented here would be useful in understanding the underlying dynamics of such REMPI schemes in other molecules.

We acknowledge several useful discussions with T. Rescigno, L. A. Collins, and B. I. Schneider. Work done at the Lawrence Livermore National Laboratory, by S. N. D. and A. U. H., was performed under the auspices of the U.S. Department of Energy under Contract No. W7405-ENG-48. Work done by B. V. M. was supported by grants from the National Science Foundation (No. CHE-8521391), the Air Force Office of Scientific Research (Contract No. 87-0039), and the Office of Health and Environmental Research, U.S. Department of Energy (No. DE-FG03-87ER60513). D. L. L. also acknowledges financial support from the National Science Foundation.
'See, for example, K. Kimura, Adv. Chem. Phys. 60, 161 (1985); R. N. Compton and J. C. Miller, in Laser Applications in Physical Chemistry, edited by D. K. Evans (Dekker, New York, 1988).

${ }^{2}$ S. T. Pratt, P. M. Dehmer, and J. L. Dehmer, Chem. Phys. Lett. 105, 28 (1984).

${ }^{3}$ T. E. Sharp, At. Data 2, 119 (1971).

${ }^{4}$ S. N. Dixit, D. L. Lynch, and V. McKoy, Phys. Rev. A 30, 3332 (1984).

${ }^{5}$ S. T. Pratt, P. M. Dehmer, and J. L. Dehmer, J. Chem. Phys. 85, 3379 (1986).

${ }^{6}$ M. A. O'Halloran, S. T. Pratt, P. M. Dehmer, and J. L. Dehmer, J. Chem. Phys. 87, 3288 (1987).

${ }^{7}$ E. Y. Xu, T. Tsuboi, R. Kachru, and H. Helm, Phys. Rev. A 36, 5645 (1987).

${ }^{8}$ W. A. Chupka, J. Chem. Phys. 87, 1488 (1987)

${ }^{9}$ A. P. Hickman, Phys. Rev. Lett. 59, 1553 (1987).

${ }^{10}$ S. L. Guberman, J. Chem. Phys. 78, 1404 (1983).

${ }^{11} \mathrm{C}$. Cornaggia, A. Giusti-Suzor, and Ch. Jungen, J. Chem. Phys. 87, 3934 (1987).

${ }^{12}$ Our calculated transition moments from the $C$ state to the ${ }^{1} \Sigma_{g}\left(1 \sigma_{u}^{2}\right)$ autoionizing state are smaller than those to the ${ }^{1} \Pi_{g}$ state by a factor of approximately 10 , and thus support the dominance of the $\Pi_{g}$ autoionizing state.

${ }^{13}$ J. N. Bardsley, J. Phys. B 1, 349 (1978); 1, 365 (1978)

${ }^{14}$ A. U. Hazi, A. E. Orel, and T. N. Rescigno, Phys. Rev. Lett. 46, $918(1981)$.

${ }^{15}$ A. U. Hazi, in Electron Molecule Collisions and Photoionization Processes, edited by V. McKoy, H. Suzuki, K. Takayanagi, and S. Trajmar (Verlag Chemie International, Deerfield Beach, FL, 1983), p. 87.

${ }^{16}$ W. Domcke, C. Mundel, and L. S. Cederbaum, Comments At. Mol. Phys. 20, 293 (1987), and references therein.

${ }^{17}$ A. L. Sobolewski and W. Domcke, J. Chem. Phys. 88, 176 (1987); 88, 5571 (1988).

${ }^{18}$ R. R. Lucchese, G. Raseev, and V. McKoy, Phys. Rev. A 25, 2572 (1982).

${ }^{19}$ J. Tennyson and C. J. Noble, J. Phys. B 18, 155 (1985)

${ }^{20}$ L. A. Collins and B. I. Schneider (private communication).

${ }^{21}$ D. J. Malik, J. Eccles, and D. Secrest, J. Comput. Phys. 38, 157 (1980).

${ }^{22}$ S. N. Dixit and V. McKoy, J. Chem. Phys. 82, 3546 (1985).

${ }^{23}$ S. T. Pratt, M. A. O'Halloran, P. M. Dehmer, and J. L. Dehmer, in Multiphoton Processes, edited by S. J. Smith and P. L. Knight (Cambridge Univ. Press, Oxford, 1988), p. 137.

${ }^{24}$ D. R. Bates, J. Chem. Phys. 19, 1122 (1951). 